\title{
Endoscopic supraorbital eyebrow approach for the surgical treatment of extraaxial and intraaxial tumors
}

\author{
Roberto Gazzeri, M.D., ${ }^{1,2}$ Yuya Nishiyama, M.D., Ph.D., ${ }^{1,3}$ and Charles Teo, M.D. ${ }^{1}$ \\ ${ }^{1}$ Centre for Minimally Invasive Neurosurgery, Prince of Wales Private Hospital, Sydney, Australia; \\ ${ }^{2}$ Department of Neurosurgery, San Giovanni Addolorata Hospital, Rome, Italy; and ${ }^{3}$ Department of \\ Neurosurgery, Fujita Health University School of Medicine, Toyoake, Japan
}

\begin{abstract}
Object. The supraorbital eyebrow approach is a minimally invasive technique that offers wide access to the anterior skull base region and parasellar area through a subfrontal corridor. The use of neuroendoscopy allows one to extend the approach further to the pituitary fossa, the anterior third ventricle, the interpeduncular cistern, the anterior and medial temporal lobe, and the middle fossa. The supraorbital approach involves a limited skin incision, with minimal soft-tissue dissection and a small craniotomy, thus carrying relatively low approach-related morbidity.

Methods. All consecutive patients who underwent the endoscopic supraorbital eyebrow approach were retrospectively analyzed for lesion location, pathology, length of stay, complications, and cosmetic results.

Results. During a 56-month period, 97 patients (mean age 58.5 years) underwent an endoscopic eyebrow approach to resect extra- and intraaxial brain lesions. The most common pathologies treated were meningiomas $(\mathrm{n}=$ 41); craniopharyngiomas $(n=22)$; dermoid tumors $(n=7)$; metastases $(n=4)$; gliomas $(n=3)$; and other miscellaneous frontal, parasellar, and midbrain $(n=23)$ lesions. The median length of postoperative hospital stay was 2.7 days (range 1-8 days). In 82 patients a total removal of the lesion was performed, while in 15 patients a near-total or subtotal removal was achieved. There were no postoperative hematomas, cerebrospinal fluid leaks, or severe neurological deficits, with the exception of 2 cases of visual deterioration and 1 case each of meningitis, stroke, and third cranial nerve paresis. Other complications directly related to the approach included 2 cases of skin burn as a direct result of heat transmission from the microscope light, 1 case of right frontal palsy, 2 cases of frontal numbness, and 1 case of bone remodeling 1 year after surgery.

Conclusions. The endoscopic supraorbital eyebrow approach is a safe and effective minimally invasive approach to remove extra- and intraaxial anterior skull base, parasellar, and frontal lesions, promoting a rapid recovery and short hospital stay. The location of the eyebrow incision demands a meticulous cosmetic closure, but, with proper technique, cosmetic results are excellent.

(http://thejns.org/doi/abs/10.3171/2014.7.FOCUS14203)
\end{abstract}

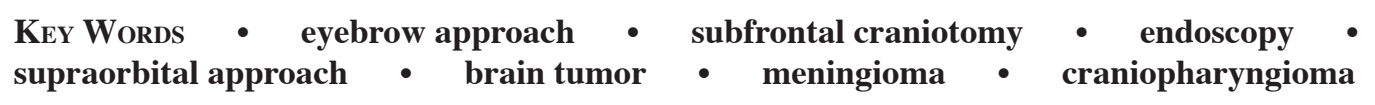

$\mathrm{T}$ He keyhole supraorbital eyebrow craniotomy provides access to lesions located in the parasellar region, cavernous sinus, orbital fissure, anterior and posterior cerebral circulation, and orbit..$^{15,19,20,22}$ In contrast to a pterional approach, it does not require substantial temporalis muscle mobilization, but controversy exists regarding whether this technique may afford satisfactory surgical exposure when compared with conventional approaches to the anterior cranial fossa. The potential advantages of the minimally invasive eyebrow craniotomy are related to shorter surgical procedures and hospital stays, less operative trauma, improved relief from pain, and better cosmetic outcomes. The simplicity and versatility of the eyebrow supraorbital approach make it one of the most efficient skull base techniques. Therefore, this

Abbreviations used in this paper: DNET $=$ dysembryoplastic neuroepithelial tumor; VASC $=$ visual analog scale for cosmesis. study was performed to document the effectiveness and safety of endoscopy during the eyebrow approach for the management of extra- and intraaxial tumors.

\section{Methods}

The medical records, clinical notes, and imaging studies of all patients who underwent an endoscopeassisted eyebrow approach performed between January 2006 and 2010 by the senior author (C.T.) were reviewed retrospectively.

To evaluate whether this approach provided adequate accessibility and exposure to all these lesions, we reviewed inpatient and outpatient records and recorded the following for analysis: age, sex, radiological findings, surgical details, length of postoperative hospital stay, complications, and long-term outcome. In all cases, for precise preoperative planning, high-resolution brain MRI 
and/or head CT was carried out. The eyebrow approach was only performed when the analysis of the preoperative images depicted clearly that complete and safe debulking of the lesion could be achieved, allowing a maximally effective yet minimally invasive surgical therapy. Tumor characteristics were recorded, including maximal diameter, location, presence of cavernous sinus and optic canal invasion, and vascular encasement of the carotid artery as well as the anterior cerebral artery complex. Early postoperative contrast-enhanced MRI was performed in all cases within 48 hours after surgery for malignant lesions and approximately 6 weeks postoperatively for benign lesions, to look for residual tumor.

Resection rates for tumors were considered as follows: gross-total removal, when no residual tumor was visible on postoperative MR images; near-total removal, when $>90 \%$ of the lesion was resected; and subtotal removal, when $<90 \%$ of tumor was removed.

At follow-up all patients underwent a complete neurological examination including functional testing of facial and frontal nerves. The cosmetic result was judged by visual inspection. The subjective satisfaction of the wound appearance was measured using a visual analog scale for cosmesis (VASC). Patients were asked by a neurosurgeon not involved in the surgery for a cosmetic assessment of their wounds at the 12-month follow-up visit. A VASC, ranging from "least satisfied" (score of 0 ) to "most satisfied" (score of 100), was completed by each patient. Enhanced MRI was performed at 6, 12, and 24 months of follow-up in all cases to look for recurrent tumor.

\section{Surgical Technique}

After endotracheal intubation and general anesthesia are achieved, the patient is placed supine in the $20^{\circ} \mathrm{re}-$ verse Trendelenburg position to facilitate venous drainage. The head is fixed in a 3-pin Mayfield headholder and positioned with $20^{\circ}$ of neck extension to allow the frontal lobe to auto-retract, falling away from the anterior cranial fossa floor. According to the location of the pathology, the degree of head rotation toward the contralateral side is determined: a $15^{\circ}-30^{\circ}$ head rotation for ipsilateral lesions and a $45^{\circ}-60^{\circ}$ rotation for lesions of the anterior fossa, olfactory groove, and contralateral lesions. Once the patient is positioned, frameless stereotactic guidance helps determine the surgical trajectory and to map the location of the frontal sinus.

A temporary tarsorrhaphy suture is placed to protect the cornea and sclera. The brow is not shaved. Although there are several options when making the initial skin incision, we prefer to make the incision within the eyebrow at its most superior margin, avoiding a direct cut across the hair follicles.

To avoid frontal numbness from injury to the supraorbital nerve, the supraorbital notch is the medial limit of the skin incision. The incision is then carried to the lateral margin of the eyebrow. It is rarely necessary to extend the incision beyond the eyebrow, but in cases in which more scalp retraction is needed, we have carried the incision up to $1 \mathrm{~cm}$ more laterally, maintaining a cosmetically pleasing result. At this point, extensive subgaleal dissection will improve retraction superiorly. The supraorbital nerve is carefully dissected and preserved. The scalp is retracted with fish hooks superiorly. The frontalis muscle is incised in the line of the incision. An inferiorly based U-shaped pericranial flap is cut and elevated. The temporalis fascia is opened to expose the keyhole region: a 4-mm rough diamond bur is used with an air-powered drill to create a small bur hole to expose the frontal lobe dura (Fig. 1A). In situations in which more superior visualization is required, the superior orbital rim may be removed in one piece along with the frontal craniotomy.

The supraorbital craniotomy is $2-3 \mathrm{~cm}$ wide and $1.5-2 \mathrm{~cm}$ high. The craniotomy is large enough to accommodate fully opened bipolar forceps. When the supraorbital foramen/notch is lateral to the frontal sinus, it serves as the inferomedial limit to the craniotomy. Otherwise, the craniotomy is taken to the margin of the frontal sinus without violating it. Frameless stereotactic guidance is helpful in localizing the frontal sinus. The base of the craniotomy is taken as low as possible and parallel to the orbital rim. To improve visualization, the inner cortical margin of the frontal bone and the bony ridges of the orbital roof are drilled flat extradurally using a high-speed drill (Fig. 1B).

The dura is opened in a U-shaped fashion with its flap based toward the cranial base and is fixed inferiorly with 2 sutures. No brain retractors are used. The first intradural step is wide opening of the proximal sylvian fissure and the prechiasmatic, opticocarotid, and carotid-oculomotor cisterns for CSF release and brain relaxation. These latter 3 corridors are the primary working windows of this subfrontal approach. Dissection of arachnoid at the base of the frontal lobe and within the sylvian fissure frees the frontal lobe from the skull base and temporal lobe, allowing it to fall away with gravity.

After the pathology is addressed as thoroughly as possible with the microscope, a handheld $30^{\circ} 4$-mm-diameter rigid endoscope (Aesculap AG) is placed into the working space to enhance the surgeon's view. The endoscope is advanced in a straight line toward the opticocarotid complex, keeping the shaft of the endoscope stabilized against the edge of the craniotomy. The endoscope is rotated without displacement of the shaft to inspect the hidden angles. By aiming the angled endoscope laterally, medially, superiorly, or inferiorly, a comprehensive view of the entire anterior cranial fossa floor may be obtained. To obtain each view, the endoscope is adjusted by rotating the rod lens and camera to keep the image upright and the vector of the view ideal. If the tumor is clearly visible with the microscope, the endoscope is simply used to assess the competency of removal at the end of the procedure. If residual tumor is seen only with the endoscope, subsequent removal is performed under endoscopic control, usually with angled instrumentation (Fig. 2).

After the pathology of interest is addressed, the dura is closed in a watertight fashion and is hitched inferiorly to reduce the extradural space created by drilling of the orbital roof. The bone flap is fixed with 2 titanium Craniofix plates (Aesculap AG). Demineralized bone matrix may be used to fill in the craniotomy line. Muscular and subcutaneous layers are closed with interrupted sutures. The skin is reapproximated with 3-0 nylon subcuticular 


\section{Endoscopic supraorbital eyebrow approach}

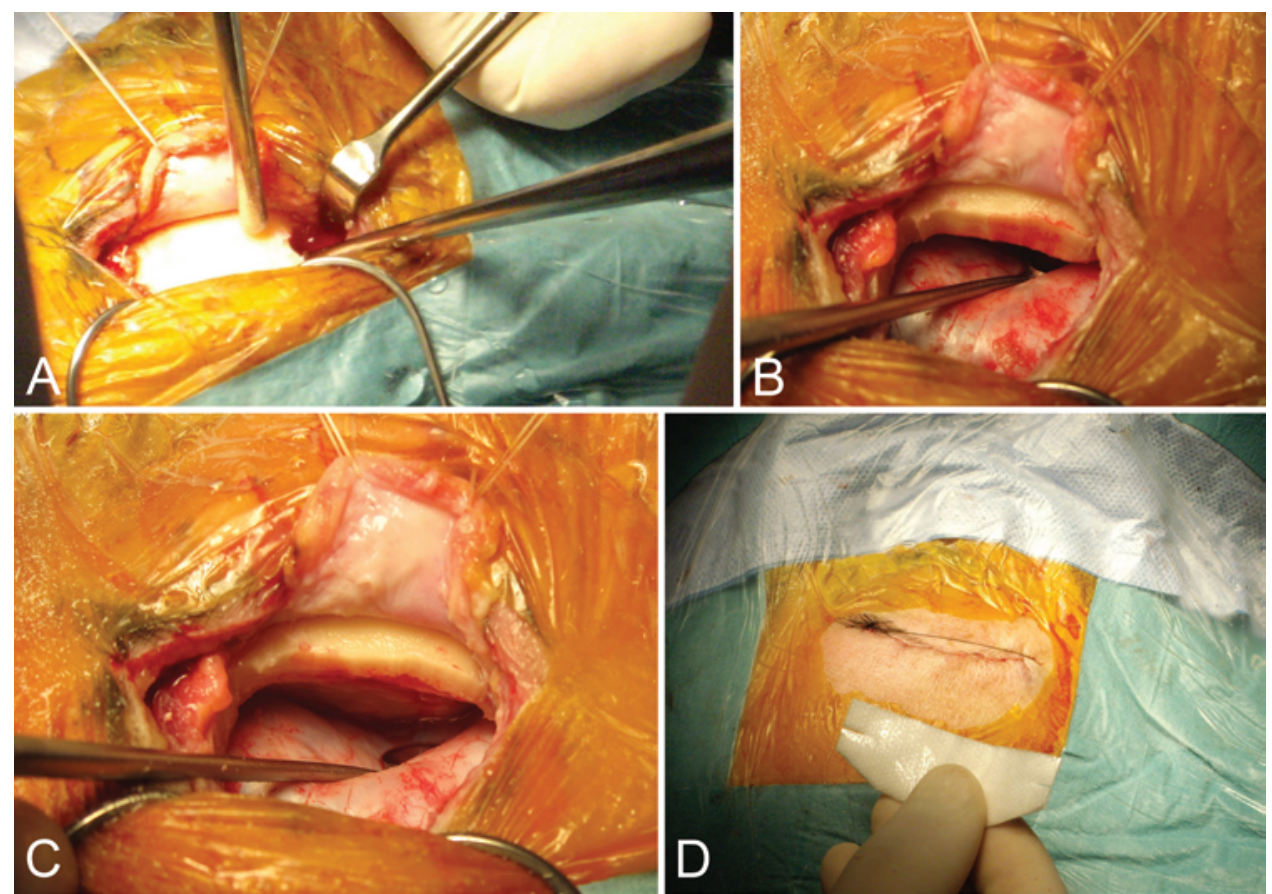

FIG. 1. Intraoperative photographs. A: Exposure of the initial craniotomy that is removed using 1 small bur hole below the superior temporal line, underneath the temporalis muscle, avoiding penetration of the orbit. Craniotomy should avoid the frontal sinus. B and C: The inner bone edges of the orbital rim are drilled flat extradurally using a high-speed drill. Removal of the inner edge can significantly increase the angle for visualization and manipulation. D: At closure, muscular and subcutaneous layers are closed with interrupted absorbable sutures, and skin edges are reapproximated with intracutaneous sutures.

closure without knots. The loose ends of this closure are tied over a nonadherent perforated plastic film dressing. After undraping, pressure is applied over the incision with gauze until the patient is extubated and breathing comfortably to prevent formation of a pseudomeningocele. The suture is removed on the 5 th postoperative day.

\section{Results}

\section{Patient Population}

A total of 97 patients (32 men and 65 women) who met the inclusion criteria were identified; their ages ranged from 10 to 75 years (mean age 58.5 years).

Follow-up periods ranged from 24 months to 7 years (mean 38 months). The most common pathology was meningioma, found in 41 patients $(42.2 \%)$, followed by craniopharyngioma in 22 patients $(22.7 \%)$. Pathology data are summarized in Table 1. Meningiomas were found most commonly in the anterior clinoid process (14 cases), tuberculum sellae (12 cases), and cavernous sinus ( 8 cases) (Table 2). In 3 cases of intraaxial tumor, the histological diagnosis was glioma, and in 3 other cases it was metastases. Five patients with incomplete tumor removal (1 lowgrade astrocytoma and 4 craniopharyngiomas) underwent previous standard craniotomy performed at another hospital. In 4 cases ( 1 metastasis and 3 hemangiomas) the lesions were located in the retro-orbital compartment, in proximity to the orbital roof and apex. In these cases, complete debulking was achieved by removing the orbital rim and orbital roof and incising the periorbita. In the remaining 93 cases the orbital rim was not removed, but the ridges on the orbital roof were drilled down to achieve a flat trajectory. Three cases of ventral midbrain lesions ( 2 cavernomas and 1 pilocytic astrocytoma) were treated using a contralateral eyebrow approach, which provided a better angle of attack, with access to cerebral peduncles by operating with the endoscope through the window between basilar perforators.

Seventy-three patients had a preoperative visual compromise. After surgical decompression, improvement of visual field defects or visual acuity was observed in 57 patients. Visual deficits remained unchanged in 14 patients and worsened in only 2 patients. The median postoperative length of stay was 2.7 days (range 1-8 days), with a typical subintensive care unit stay of 1 day. No patient underwent radiosurgery or standard radiotherapy after surgery.

Postoperative MRI, performed in most cases at 6 weeks, revealed complete tumor removal in 82 cases $(84.5 \%)$, near-total removal in 8 cases $(8.2 \%)$, and subtotal removal in 7 cases (7.2\%) (Figs. 3 and 4). Near-total removal was achieved for 3 low-grade astrocytomas, for 2 cavernous sinus meningiomas, for 1 craniopharyngioma, and for 2 dermoid tumors. Subtotal removal was achieved in 5 cases of cavernous sinus meningiomas, in case of 1 craniopharyngioma, and in 1 case of optic meningioma.

\section{Complications}

No intraoperative complications occurred, and remodeling of the craniotomy (conversion to a larger craniotomy) was never necessary. No patient experienced retractorrelated edema or intraparenchymal hematoma. Although 

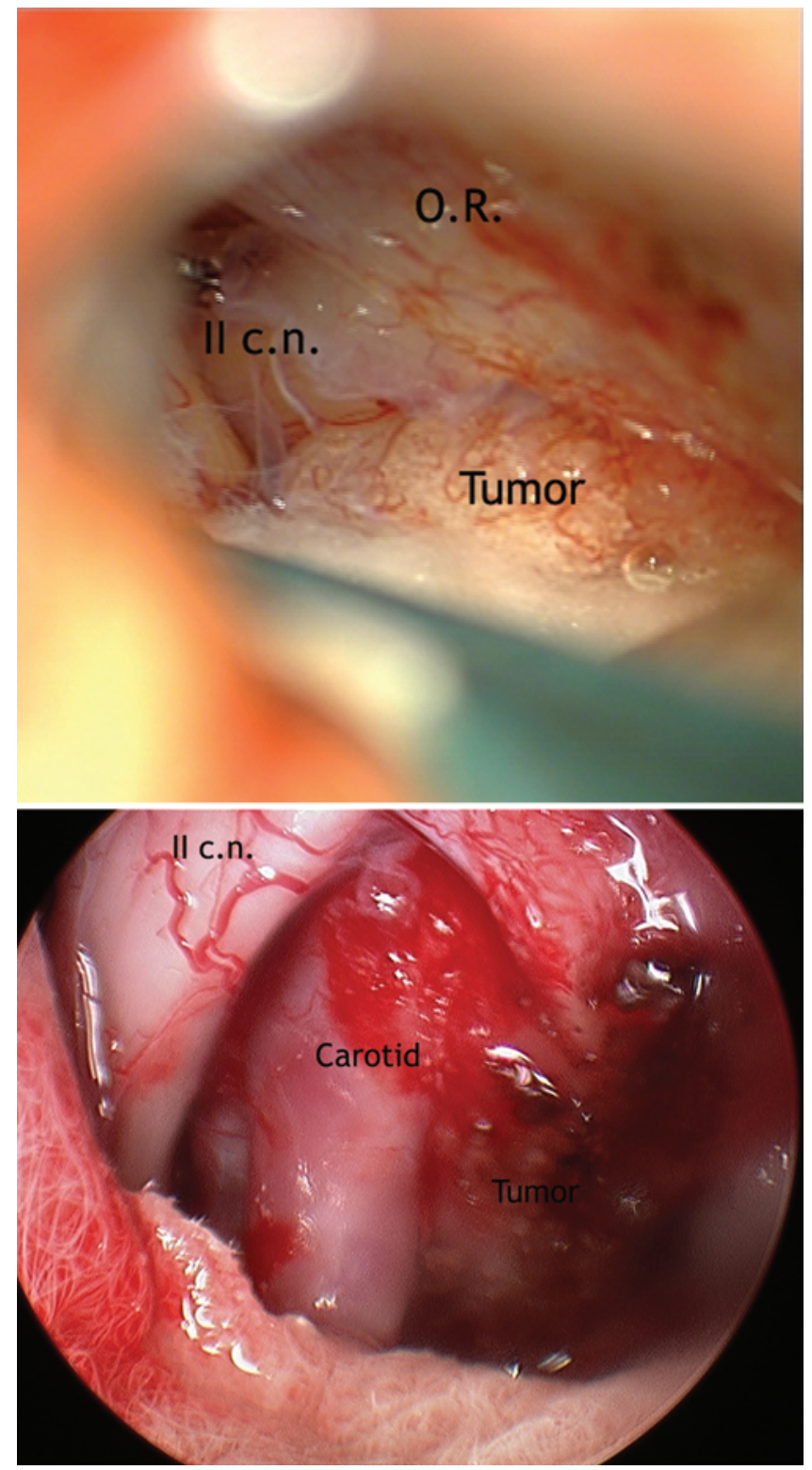

Fig. 2. Upper: Intraoperative view from the operative microscope showing the parasellar area during a supraorbital approach to an anterior clinoid meningioma. Lower: A $30^{\circ}$ endoscopic image of the suprasellar region after subtotal tumor removal, with remnants of the meningioma. Note the endoscopic appearance of the optic nerve and carotid artery, compared with the microscopic visualization. c.n. = cranial nerve; O.R. = orbital rim.

in 4 cases the craniotomy breached the lateral edge of the frontal sinus, no patient developed postoperative rhinorrhea. The most common complication was postoperative panhypopituitarism in 4 patients after craniopharyngioma removal. Ten patients with craniopharyngioma and 1 with a Rathke cleft cyst had preoperative panhypopituitarism requiring hormone replacement therapy. Of the 15 patients (12 harboring craniopharyngiomas and 2 harboring Rathke cleft cysts) without panhypopituitarism, new postoperative anterior hormonal loss occurred in 4 patients, although 3 of these 4 patients had some degree of preexisting hypopituitarism ( 2 cases of adrenocorticotropic hormone deficiency
TABLE 1: Extraaxial and intraaxial pathologies treated using the endoscope-assisted supraorbital eyebrow approach

\begin{tabular}{lc}
\hline \multicolumn{1}{c}{ Pathology } & No. of Patients \\
\hline meningioma & 41 \\
craniopharyngioma & 22 \\
dermoid tumor & 7 \\
astrocytoma Grade II & 3 \\
hemangioma & 6 \\
pituitary adenoma & 3 \\
metastasis & 4 \\
Rathke cleft cyst & 3 \\
DNET & 2 \\
cavernous angioma & 1 \\
juvenile pilocytic astrocytoma & 1 \\
hamartoma & 1 \\
teratoma & 1 \\
neurenteric cyst & 1 \\
granular cell tumor & 1 \\
\hline
\end{tabular}

and 1 case of adrenocorticotropic hormone and growth hormone deficiencies). Five patients (4 with craniopharyngiomas and 1 with a Rathke cleft cyst) with partial anterior pituitary (thyrotropic and gonadotropic) insufficiency after surgery received initial hormone replacement therapy with levothyroxine and sex steroids. None of these 5 patients at last follow-up required additional correction of the hormone deficiencies. Postoperative weight gain was observed in 3 patients with large craniopharyngiomas growing inside the cavity of the third ventricle. Visual deficits worsened in 2 patients ( 1 craniopharyngioma and 1 cavernous sinus meningioma), although severe visual impairment was present before surgery. One patient treated for cavernous angioma removal in the septal area close to the optic tract suffered a small stroke in the head of the caudate nucleus. One patient treated with a combined supraorbital and endonasal approach for an anterior cranial fossa meningioma suffered meningitis due to Escherichia coli 7 days after hospital discharge and required 2 weeks of intravenous antibiotics. Complications observed in the series are summarized in Table 3.

In our series of patients, there were no severe complications directly related to the supraorbital approach, with the exception of 2 cases of full and partial thickness burns as a direct result of heat transmission from having

TABLE 2: Anterior skull base meningiomas treated with the supraorbital approach

\begin{tabular}{cc}
\hline Meningioma Location & No. of Patients \\
\hline anterior clinoid & 14 \\
tuberculum sellae & 12 \\
cavernous sinus & 8 \\
sphenoid wing & 5 \\
olfactory groove & 1 \\
optic & 1 \\
\hline
\end{tabular}



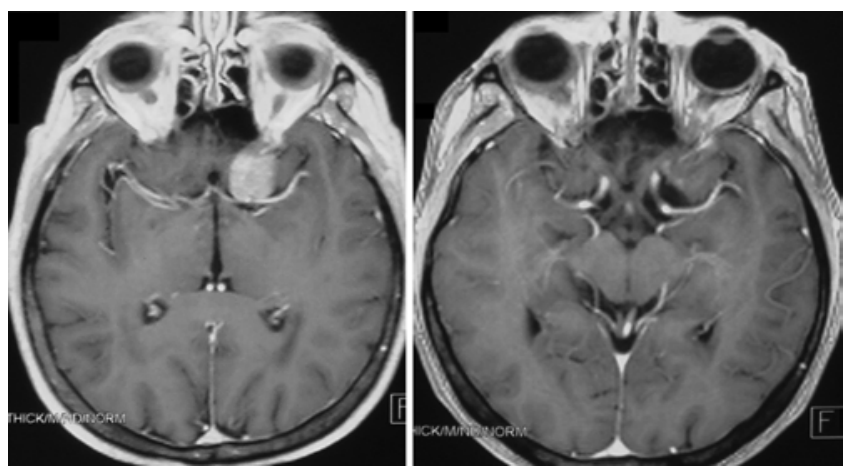

FIG. 3. Preoperative and postoperative T1-weighted axial images obtained in a 57-year-old patient who had an anterior clinoid meningioma. Left: Preoperative image revealing a solid homogeneous tumor incorporating the internal carotid artery bifurcation. Right: Postoperative image showing complete tumor removal.

the microscope light on at maximum intensity. Once the senior surgeon (C.T.) realized the problem and modified the settings (never greater than $75 \%$ ), there have not been any cases of burns. Other minor complications included 1 case of right frontal palsy, 2 cases of frontal numbness, and 1 case of bone remodeling at 1 year of follow-up. No patient developed postoperative CSF leaks.

\section{Follow-Up}

At 2 years of follow-up, MRI imaging studies showed tumor recurrence or progression in 8 cases $(2$ cavernous sinus meningiomas, 2 craniopharyngiomas, 1 optic meningioma, 1 granular cell tumor, 1 dysembryoplastic neuroepithelial tumor [DNET], and 1 astrocytoma Grade II). Tu- mors recurred in patients who had undergone near-total or subtotal removal. Three patients with astrocytoma Grade II underwent a near-total tumor removal $(>90 \%)$ and received no other treatment; all but 1 patient had no evidence of tumor growth on their most recent MRI studies $(24,38$, and 45 months, respectively, after surgery).

Of the 3 patients with metastatic carcinoma, 1 patient died of systemic disease progression 15 months after surgery. Reoperation was performed in 2 patients for recurrence of cavernous sinus meningioma and in 1 patient for a DNET.

For the cosmetic outcome measurement, 94 patients (96.9\%) were satisfied with the cosmetic result of the supraorbital eyebrow approach, with a score of greater than 85 on the VASC. Two patients suffered from skin burns (1 minor and 1 major) due to heating from excessive illumination of xenon light from the operative microscope; these two patients had VASC scores of 75 and 69, respectively. One patient suffered from bone flap remodeling 12 months after surgery, with a VASC score of 65.

\section{Discussion}

Many different techniques to treat lesions of the anterior and middle cranial fossae using a subfrontal route have been well described during the past decades. . $^{1,3,6,8,17,22}$ Modern surgical series of cranial base meningiomas, craniopharyngiomas, and pituitary macroadenomas report varying rates of resection that use a variety of approaches, typically pterional, subfrontal, or bifrontal. The keyhole concept has been developed in the past years..$^{11,17,18,22}$ It is based on an important principle: smaller openings create
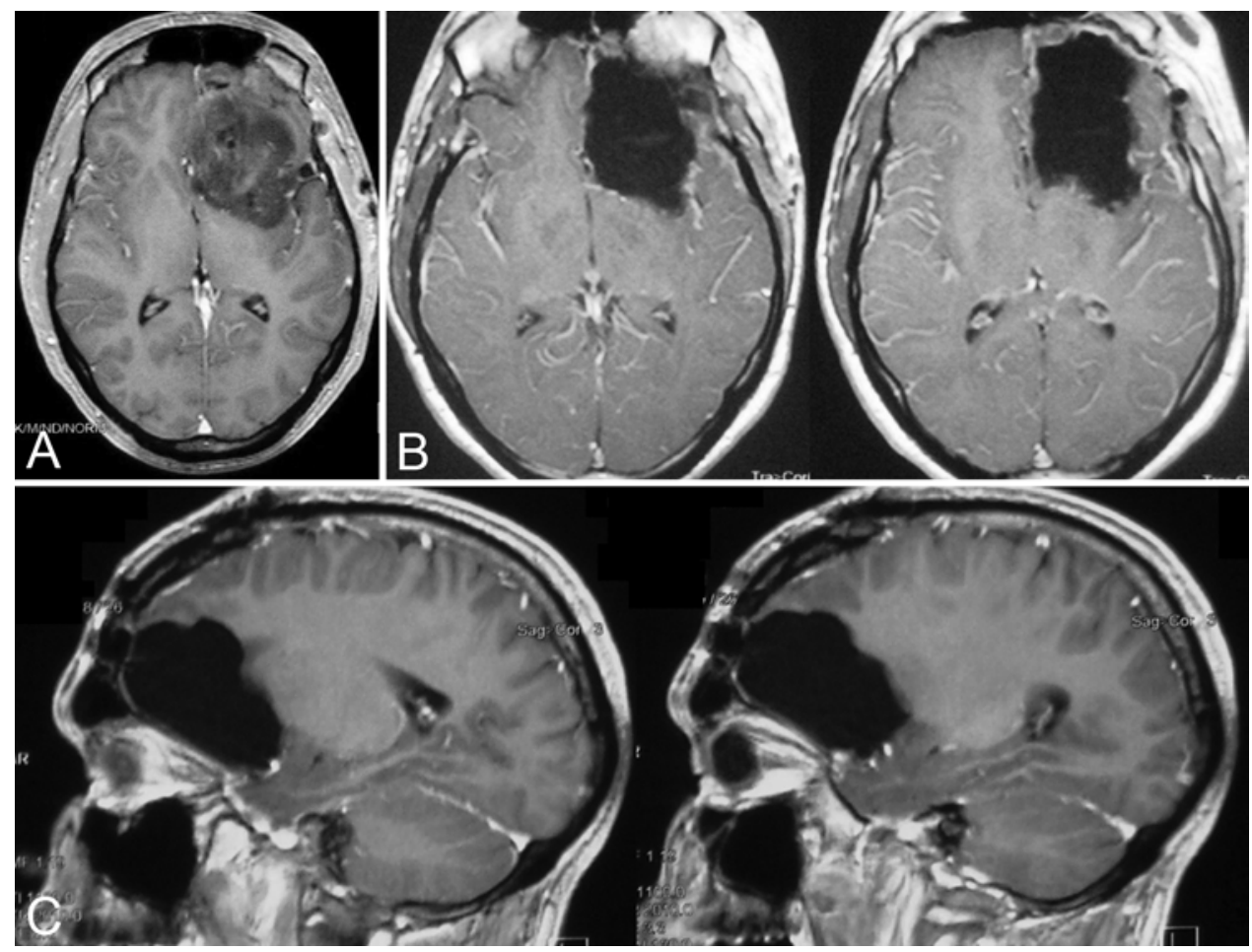

FIG. 4. Preoperative (A) and 2-year postoperative axial (B) and sagittal (C) Gd-enhanced T1-weighted MR images obtained after removal of a Grade II left frontal astrocytoma. 
TABLE 3: Postoperative complications

\begin{tabular}{lc}
\hline \multicolumn{1}{c}{ Complications } & No. of Patients \\
\hline transient partial hypopituitarism & 5 \\
hypopituitarism & 4 \\
diabetes insipidus & 4 \\
transient diabetes insipidus & 3 \\
obesity & 3 \\
amenorrhea & 1 \\
visual worsening & 2 \\
meningitis (E. coli) & 1 \\
stroke & 1 \\
cranial nerve III palsy & 2 \\
skin burn* & 2 \\
frontal numbness & \\
right frontal palsy* & 2 \\
bone flap remodeling & 1 \\
\hline
\end{tabular}

* Complications directly related to the supraorbital approach.

a surgical field that widens as the distance from the craniotomy increases. The achievement of a smaller opening is made possible by performing the craniotomy in a strategic location that takes advantage of the regional anatomy to focus the opening on the lesion of interest. Instead of a large frontotemporal craniotomy, a small craniotomy can be used according to the concept of keyhole surgery, as described by Perneczky et al., ${ }^{11}$ which is a minimally invasive approach to the anterior cranial fossa including the sellar and parasellar area. The eyebrow keyhole approach has recently gained popularity. Surgical series, particularly those including aneurysm cases, have been reported with satisfactory outcomes. ${ }^{3,6,12,20}$

The anatomies of the parasellar and suprasellar regions lend themselves more favorably to an anterior approach. This region is bordered by the mesencephalon posteriorly and the mesial portions of the temporal lobe laterally. Approaches from the sides require considerable brain retraction or alternatively a wider splitting of the sylvian fissure. Conversely, approaches from the anterior direction offer several working windows without the need for significant brain retraction. The choice of any surgical approach should be based mainly on the anatomical exposure that it provides; although a bicoronal incision and a bifrontal or frontal craniotomy provide adequate exposure of the anterior skull base, in most cases they also unnecessarily expose large surface areas of brain that are not critical to the exposure of the lesion itself.

Experience and familiarity with using a particular approach are also important points to consider. However, the study by Figueiredo et al. demonstrated that less invasive openings may offer similar surgical working areas to those provided by standard craniotomies and constitute excellent alternatives to the pterional craniotomy in selected cases..$^{10} \mathrm{~A}$ review of the literature showed much research addressing temporalis muscle atrophy, chewing problems, mandibular pain, scalp alopecia, and numbness around the incision of a pterional craniotomy. $7,10,13,21$
The supraorbital eyebrow craniotomy is an anterolateral approach that allows the surgeon to address diverse pathology of the anterior cranial fossa, parasellar region, proximal sylvian fissure, ipsilateral and contralateral regions of the circle of Willis, basal frontal lobe, and ventral brainstem. When supplemented with intracranial endoscopy, lesions of the lateral cavernous sinus, pituitary fossa, contralateral circle of Willis, and ipsilateral retroorbital space may be addressed. Meningioma of the anterior or middle cranial base was the most common tumor type in our series, and benign tumors in these regions accounted for most of the abnormalities in this series.

The supraorbital approach provides the most direct surgical trajectory to the frontal pole and some mesial and orbital parts of the frontal lobe (with no need for fixed brain retraction) as well as to orbitofrontal and skull base lesions and those in the mesial temporal lobe. In cases of an approach to mesial temporal lesions, tailored head elevation and rotation facilitates venous drainage offering an ergonomic working position with optimal exposure. ${ }^{19}$

The size of the frontal sinus must be considered in planning the supraorbital approach: in cases of frontal sinus breaching there is an increased risk of CSF rhinorrhea and infection. Moreover, a large frontal sinus displaces the craniotomy laterally, altering the operative trajectory. To avoid unnecessary frontal sinus breaches and to choose the surgical trajectory prior to making the skin incision, we performed the surgical procedure using neuronavigation in all our cases; frameless image guidance was helpful in mapping the frontal sinus and planning the craniotomy lateral to the lateral-most edge of the sinus. We did not offer the supraorbital approach as an option to patients with large frontal sinuses, but this was only an issue in few cases. In our study, the craniotomy breached the lateral edge of the frontal sinus in 4 patients. When the frontal sinus was entered, the mucosa was stripped and the sinus was packed with Betadine-soaked Gelfoam. The small surgical opening was carefully repaired with fat or muscle and was covered with vascularized pericranial flap overlay.

Visualization of lesions and surrounding anatomy using the supraorbital approach may be enhanced by endoscopy. ${ }^{9}$ The advantages of endoscopy include more direct illumination in a deep operative field and the ability to achieve an angled line of sight to view previously hidden regions. ${ }^{4,5,12,14,16}$ The $0^{\circ}$ angled endoscope provides a view of the operative field with higher magnification and more direct illumination than the operating microscope. In our series, the endoscope was used for tumor resection in the majority of cases; the endoscope was used for inspection in only a few cases. We usually performed the initial tumor exposure and resection under microscopic visualization. We then used the endoscope to selectively extend the field of view and have better access to the pituitary fossa, the region under the ipsilateral optic nerve, the interpeduncular cistern, the anterior third ventricle, and the anterior interhemispheric fissure. The endoscope allowed us to identify residual tumor and important surrounding neurovascular structures once the tumor had been partially removed or, as in the case of cystic craniopharyngiomas, once the tumor had been decompressed by cyst 
drainage. It is imperative to perform endoscopic exploration earlier in the operation rather than later to better visualize tumor adherence to neurovascular structures poorly seen by the operative microscope. Tumor removal under endoscopic visualization can be performed using a 3-hand technique, in which one surgeon drives the endoscope and the other surgeon performs a bimanual microsurgical tumor removal.

Suprasellar portions of a sellar tumor can be removed by splitting the fissure and using a $30^{\circ}$ endoscope for visualization. Removal of intrasellar portions can be augmented using the $30^{\circ}$ endoscope rotated the other way, aimed inferiorly into the often capacious sella. The superior view afforded by the $30^{\circ}$ scope has revealed, on several occasions, tumor carpeting the parasellar portion of the internal carotid arteries, the undersurface of the optic apparatus, the interpeduncular fossa, and the pituitary stalk. Although our surgical goal was total removal of the lesions, in patients with extensive tumor growth in the optic canals or cavernous sinuses, tumor adhesions to the optic nerve, pituitary stalk, and vascular structures, attempts at removing these tumor remnants were limited to minimize the risk of postoperative neurological deficits: in these cases small tumor remnants were left behind.

With the use of the endoscope, the interchiasmatic, opticocarotid, and carotid-oculomotor cisterns are the 3 fundamental corridors and primary working windows from the subfrontal approach. Although the eyebrow approach offers access to most of the anterior skull base, there are a few areas that may be difficult to reach, for example, the midline depression of the olfactory groove along the anterior cranial fossa floor that lies below the line of sight provided by the operating microscope. This blind spot may be reachable only with the use of an angled endoscope and angled instrumentation. Endoscopy is also fundamental to visualize the anterior midline extent of olfactory and planum meningiomas. ${ }^{9}$ In cases of midline tumors that do not extend beyond the midpoint of the orbits, a standard endonasal transsphenoidal and transethmoidal approach may be preferable. In cases of huge anterior skull base meningiomas with bilateral orbital roof extension, we prefer a mini-bifrontal craniotomy through an approximately $6-\mathrm{cm}$ incision just in front of the hairline.

In many cases of craniopharyngiomas, the thinly stretched pituitary stalk was visible only with the $30^{\circ}$ angled endoscope. The endoscope, with its higher magnification, better observation, and increased illumination, can provide information that may not be available with the microscope. It can "look around corners" and allow observation of areas considered blind under the microscope with a clear depiction of details in close-up positions. ${ }^{4,5,12,14,16}$ Fatemi et al. reported on 13 patients who underwent a supraorbital approach to treat craniopharyngioma and tuberculum sellae meningiomas; in $31 \%$ of their cases, the endoscope was used to augment the surgical working area. ${ }^{9}$

In our study, the endoscope was useful in gaining an appreciation of the anatomy in the majority of cases and provided better visualization of those areas poorly visualized with the microscope alone in all cases. Offering superior illumination with a wide panoramic view of the surgical target, the endoscope allowed a direct view of the optic nerves and optic canals bilaterally, thus facilitating a safe extracapsular dissection of the tumors off the visual apparatus with preservation of the vascular structures and pituitary stalk. In cases of lesions intimately involved with critical neurovascular structures in which different angles of view are required, the use of the angled endoscope allowed us to fully visualize these structures and to assess for tumor remnants that were out of the view of the microscope. Furthermore, the use of the endoscope and angled instruments allowed for removal of intracanalicular extension of all tuberculum sellae meningiomas. When the target pathology is deep seated as in the case of ventral midbrain or third ventricle lesions, we perform surgery with the endoscope as the only source of visualization. We treated 3 ventral brainstem lesions using a contralateral eyebrow approach, which provided a better angle of attack with access to cerebral peduncles by operating with the endoscope through the window between basilar artery perforators.

Generally, the first-line therapy for the treatment of gliomas and brain metastases is surgical resection. The aim in these patients is to minimize normal brain exposure and surgical approach morbidity. The eyebrow craniotomy is the most direct route for a transcortical approach to tumors of the frontal pole and orbitofrontal surface.

In our experience, the most common complication was transient forehead numbness from retraction injury to the supraorbital nerve. Although a common complaint in the early postoperative period, this symptom was rarely permanent. We also found frontalis weakness in the early postoperative phase, presumably from stretching of the frontalis branch of the facial nerve. This was also temporary in the majority of cases. In a series by Reish and Perneczky the authors reported a $7.5 \%$ incidence of transient numbness and a $5.5 \%$ incidence of permanent frontalis weakness. ${ }^{19}$ Meticulous planning of the skin incision and careful softtissue dissection may avoid injury to the frontalis branch of the facial nerve. We extended the skin incision more laterally than the limit of the eyebrow in 6 cases only; limiting the lateral extension of the skin incision can diminish the frequency of frontalis palsy due to inadvertently sectioning the frontalis branch of the facial nerve, which is difficult to identify. In one case we had a right frontal palsy probably due to a more lateral skin incision.

Skin burns from heat caused by the energy of the light of the operative microscope have been reported, even when xenon light sources are used. ${ }^{2}$ In cases of higher magnification, higher light intensity is required, thus generating more heat. As light propagates through the layers of tissues, it loses intensity and heat. The skin, as the first layer of tissue and that closest to the microscope's light, is most vulnerable. The risk of skin injury increases with longer exposure time and shorter distance from the light source. In our series 2 patients suffered eyebrow skin burns caused by the high intensity of the light of the operative microscope. We suggest that avoiding maximum intensity of the light or using it for as short a time as possible will minimize potential damage to the skin of the eyebrow area. 
There are several limitations of our study that have to be taken into account when interpreting the results. First, this study is a retrospective analysis of various benign and malignant intracranial lesions. Furthermore, long-term patient outcome and recurrence rate in benign tumors with subtotal or near-total removal were not analyzed due to a short follow-up. Another limitation of this study is the absence of direct comparison with more traditional craniotomies. The results also reflect a personal preference of the senior author (C.T.) regarding the choice of this approach. Further investigations with longer follow-up periods and prospective trials comparing different craniotomies and approaches are recommended.

\section{Conclusions}

The supraorbital eyebrow approach to the anterior cranial base is an efficient and safe method for the treatment of intra- and extraaxial tumors of the anterior fossa floor, anterior frontal lobe, and parasellar region. It involves a limited skin incision, minimal soft-tissue dissection, and a small craniotomy, thus carrying very little approach-related morbidity. Use of endoscopy further expands the surgical application of this keyhole approach to include the anterior third ventricle, the sellar and retrosellar areas, ventral brainstem, upper clivus, and even portions of the middle cranial fossa.

\section{Disclosure}

The authors report no conflict of interest concerning the materials or methods used in this study or the findings specified in this paper.

Author contributions to the study and manuscript preparation include the following. Conception and design: Gazzeri, Teo. Acquisition of data: all authors. Analysis and interpretation of data: all authors. Drafting the article: Gazzeri. Critically revising the article: Gazzeri. Reviewed submitted version of manuscript: Teo. Approved the final version of the manuscript on behalf of all authors: Gazzeri Study supervision: Teo.

\section{References}

1. Al-Mefty O: Supraorbital-pterional approach to skull base lesions. Neurosurgery 21:474-477, 1987

2. Al-Qattan MM, Clarke HM: A burn caused by the operating microscope light during brachial plexus reconstruction. $\mathbf{J}$ Hand Surg Br 19:550-551, 1994

3. Brock M, Dietz H: The small frontolateral approach for the microsurgical treatment of intracranial aneurysms. Neurochirurgia (Stuttg) 21:185-191, 1978

4. Cohen AR: Endoscope-assisted brain surgery: Part I-Evolution, basic concept, and current technique. Neurosurgery 42:224-225, 1998

5. Cohen AR: Endoscopic neurosurgery, in Wilkins RH, Rengachary SS (eds): Neurosurgery, ed 2. New York: McGrawHill, 1996, Vol 1, pp 539-546

6. Dare AO, Landi MK, Lopes DK, Grand W: Eyebrow incision for combined orbital osteotomy and supraorbital minicrani- otomy: application to aneurysms of the anterior circulation. Technical note. J Neurosurg 95:714-718, 2001

7. de Divitiis E, Esposito F, Cappabianca P, Cavallo LM, de Divitiis $\mathrm{O}$ : Tuberculum sellae meningiomas: high route or low route? A series of 51 consecutive cases. Neurosurgery 62: 556-563, 2008

8. Delashaw JB Jr, Jane JA, Kassell NF, Luce C: Supraorbital craniotomy by fracture of the anterior orbital roof. Technical note. J Neurosurg 79:615-618, 1993

9. Fatemi N, Dusick JR, de Paiva Neto MA, Malkasian D, Kelly DF: Endonasal versus supraorbital keyhole removal of craniopharyngiomas and tuberculum sellae meningiomas. Neurosurgery 64 (5 Suppl 2):269-286, 2009

10. Figueiredo EG, Deshmukh V, Nakaji P, Deshmukh P, Crusius MU, Crawford N, et al: An anatomical evaluation of the minisupraorbital approach and comparison with standard craniotomies. Neurosurgery 59 (4 Suppl 2):ONS212-ONS220, 2006

11. Fries G, Perneczky A: Endoscope-assisted brain surgery: part 2-analysis of 380 procedures. Neurosurgery 42:226-232, 1998

12. Fukushima T: Endoscope-assisted brain surgery: Part I-Evolution, basic concept, and current techniques. Neurosurgery 42:224, 1998

13. Hayhurst C, Teo C: Tuberculum sella meningioma. Otolaryngol Clin North Am 44:953-963, viii-ix, 2011

14. Hopf NJ, Perneczky A: Endoscopic neurosurgery and endoscope-assisted microneurosurgery for the treatment of intracranial cysts. Neurosurgery 43:1330-1337, 1998

15. Jallo GI, Bognár L: Eyebrow surgery: the supraciliary craniotomy: technical note. Neurosurgery 59 (1 Suppl 1):ONSE157ONSE158, 2006

16. Jallo GI, Suk I, Bognár L: A superciliary approach for anterior cranial fossa lesions in children. Technical note. J Neurosurg 103 (1 Suppl):88-93, 2005

17. Perneczky A, Fries G: Endoscope-assisted brain surgery: part 1-evolution, basic concept, and current technique. Neurosurgery 42:219-225, 1998

18. Perneczky A, Fries G: Use of a new aneurysm clip with an inverted-spring mechanism to facilitate visual control during clip application. Technical note. J Neurosurg 82:898-899, 1995

19. Reisch R, Perneczky A: Ten-year experience with the supraorbital subfrontal approach through an eyebrow skin incision. Neurosurgery 57 (4 Suppl):242-255, 2005

20. Steiger HJ, Schmid-Elsaesser R, Stummer W, Uhl E: Transorbital keyhole approach to anterior communicating artery aneurysms. Neurosurgery 48:347-352, 2001

21. Taniguchi M, Perneczky A: Subtemporal keyhole approach to the suprasellar and petroclival region: microanatomic considerations and clinical application. Neurosurgery 41:592-601, 1997

22. van Lindert E, Perneczky A, Fries G, Pierangeli E: The supraorbital keyhole approach to supratentorial aneurysms: concept and technique. Surg Neurol 49:481-490, 1998

Manuscript submitted May 10, 2014.

Accepted July 15, 2014.

Please include this information when citing this paper: DOI: 10.3171/2014.7.FOCUS14203.

Address correspondence to: Roberto Gazzeri, M.D., Department of Neurosurgery, San Giovanni Addolorata Hospital, Via Amba Aradam 9, Rome 00184, Italy. email: robertogazzeri@gmail.com. 